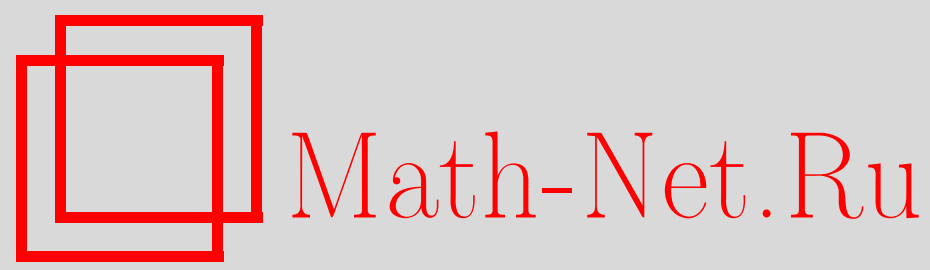

Е. Г. Скляренко, О фильтрациях в гипергомологиях, $M a-$ тем. заметки, 1997, том 61, выпуск 4, 578-582

DOI: https://doi.org/10.4213/mzm1536

Использование Общероссийского математического портала Math-Net.Ru подразумевает, что вы прочитали и согласны с пользовательским соглашением http://www . mathnet.ru/rus/agreement

Параметры загрузки:

IP : 18.209.158.208

26 апреля 2023 г., 12:59:21

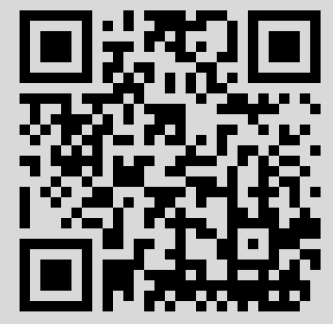


УДК 515.142.21

\title{
О ФИЛЬТРАЦИЯХ В ГИПЕРГОМОЛОГИЯХ
}

\author{
Е.Г. Скляренко
}

\begin{abstract}
В типичных ситуациях дается "внутреннее" описание гипергомологий и двух естественных фильтраций в них (не зависящее от аппарата гипергомологий).

Библиография: 11 названий.
\end{abstract}

С гипергомологиями комплекса $\mathscr{N}_{*}$ для точного слева аддитивного функтора $F$ обычно связьвают две стандартные спектральные последовательности $[1$, гл. XVII]. В неявном виде как группы гомологий или когомологий некоторых конкретных двойных комплексов гипергомологии вместе со спектральными последовательностями широко использовались в литературе, относящейся к применениям пучковых методов в теории гомологий и когомологий, и в некоторой литературе по гомологической алгебре, в частности, в монографиях [2]-[4]. Нередко (это относится, например, к [2] и [3]) при отличаюшихся подходах к описанию одних и тех же конструкций для их последующего отождествления приходится привлекать специальные аргументы, которые в конечном итоге (в терминах гипергомологий) сводятся к обоснованию независимости как самих спектральных последовательностей, так и определяющих их фильтраций, от используемых при разных подходах определяемых резольвентами Картана-Эйленберга двойных комплексов.

В настоящей заметке уточняются аргументы, подтверждаюшие инвариантность двух фильтраций в гипергомологиях (и, как следствие, двух спектральных последовательностей). Показьвается, что в двух наиболее типичных ситуациях, когда $\mathfrak{N}_{*}$ либо состоит из $F$-ацикличных объектов, либо оказьвается точной последовательностью (например, резольвентой), как сами гипергомологии, так и две естественные фильтрации в них допускают "внутреннее" описание в терминах одних только связанных последовательностей функторов без использования резольвент и других конструкций теории гипергомологий. В случае, когда $\mathscr{N}_{*}=\mathscr{C}_{*}(\mathscr{G})$ - дифференциальньй пучок цепей на топологическом пространстве таким образом возникает известная фильтрация Зимана в гомологиях.

Фильтрации в гипергомологиях комплекса $\mathscr{N}_{*}$ для функтора $F$ определяются двумя фильтрациями в биградуированном объекте $\mathscr{I}^{*} \mathscr{N}_{*}$ - подобъектами $K_{p}$ вида $\bigoplus_{i, j} \mathscr{I}^{i} \mathscr{N}_{j}$, для которых $j \leqslant p$ (первая спектральная последовательность в терминологии $\left[1\right.$, гл. XVII, §2]), и аналогичными подобъектами $K^{q}$, для которых $i \geqslant q$

Работа выполнена при поддержке Российского фонда фундаментальных исследований, грант № 96-01-01403. 
(вторая последовательность в [1]). Здесь $\mathscr{I}^{*} \mathcal{N}_{*}-$ инъективная резольвента Картана-Эйленберга комплекса $\mathscr{N}_{*}$ (см. [1, гл. XVII]). Пусть $\Phi_{p} \mathbb{H}_{m}$ и $\Phi^{q} \mathbb{H}_{m}$ - образы в гипергомологиях $\mathbb{H}_{m}\left(\mathscr{N}_{*} ; F\right)=H_{m}\left(F\left(\mathscr{I}^{*} \mathscr{N}_{*}\right)\right)$ комплекса $\mathscr{N}_{*}$ для функтора $F$ гомологий соответственно подкомплексов $F\left(K_{p}\right)$ и $F\left(K^{q}\right)$ комплекса $F\left(\mathscr{I}^{*} \mathscr{N}_{*}\right)$, рассматриваемого в стандартной полной градуировке $\bigoplus_{j-i=n} F\left(\mathscr{I}^{i} \mathscr{N}_{j}\right)$ относительно полного дифференциала $d=d^{\prime}+(-1)^{j} d^{\prime \prime}$, где $d^{\prime}, d^{\prime \prime}$ - дифференциалы, индуцированные из $\mathscr{N}_{*}$ и из $\mathscr{I}^{*}$ (см., например, [3, гл. I, п. 4.5] или [1, гл. XVII, §1]). В группах $\mathbb{H}_{m}\left(\mathscr{N}_{*} ; F\right)$ возникают две фильтрации

$$
\begin{gathered}
\mathbb{H}_{m}\left(\mathscr{N}_{*} ; F\right) \cdots \supset \Phi_{p+1} \mathbb{H}_{m} \supset \Phi_{p} \mathbb{H}_{m} \supset \cdots \supset \Phi_{m} \mathbb{H}_{m} \supset \Phi_{m-1} \mathbb{H}_{m}=0 \\
\mathbb{H}_{m}\left(\mathscr{N}_{*} ; F\right)=\Phi^{0} \mathbb{H}_{m} \supset \Phi^{1} \mathbb{H}_{m} \supset \cdots \supset \Phi^{q} \mathbb{H}_{m} \supset \cdots
\end{gathered}
$$

Для любой $F$-ацикличной резольвенты $\mathscr{C}^{*} \mathscr{N}_{*}$ Картана-Эйленберга комплекса $\mathscr{N}_{*}$ в соответствии с доказательством леммы 1.1 [5] (которое хотя и приведено там в терминах теории пучков, является категорным по духу) имеется преобразование резольвент $\alpha: \mathscr{C}^{*} \mathscr{N}_{*} \rightarrow \mathscr{I}^{*} \mathscr{N}_{*}$, единственное с точностью до гомотопии в смысле [1, гл. IV, $\left.§ 4\right]$. Преобразование $\alpha$ индуцирует изоморфизмы спектральных последовательностей обоих типов (со вторыми членами $E_{2}^{p q}$ соответственно вида $H_{-p}\left(F^{q}\left(\mathscr{N}_{*}\right)\right)$ и $\left.F^{p}\left(H_{-q}\left(\mathscr{N}_{*}\right)\right)\right)$. Поскольку фильтрации первого типа регулярны (в смысле [3, гл. I, п. 4.1]), $\alpha$ индуцирует изоморфизм фильтрованных групп первого типа $\alpha_{*}: H_{*}\left(F\left(\mathscr{C}^{*} \mathscr{N}_{*}\right)\right) \rightarrow \mathbb{H}_{*}\left(\mathscr{N}_{*} ; F\right)[3$, гл. I, теорема 4.3.1]. Преобразование $\alpha$ обеспечивает также включения $\widetilde{\Phi}^{q} \mathbb{H}_{m} \subset \Phi^{q} \mathbb{H}_{m}$, где $\widetilde{\Phi}^{q} \mathbb{H}_{m}$ - фильтрующие $\mathbb{H}_{m}\left(\mathcal{N}_{*} ; F\right)$ подгрупшы второго типа, определяемые резольвентой $\mathscr{C}^{*} \mathscr{N}_{*}$. Если резольвента $\mathscr{C}^{*} \mathscr{N}_{*}$ также инъективна, наличие гомотопически обратного к $\alpha$ преобразования резольвент обеспечивает равенства $\widetilde{\Phi}^{q} \mathbb{H}_{m}=\Phi^{q} \mathbb{H}_{m}$. Таким образом, фильтрация второго типа не зависит от выбора инъективной резольвенты $\mathscr{I}^{*} \mathscr{N}_{*}$.

В частном случае, когда $H_{*}\left(\mathscr{N}_{*}\right)=0$, имеем $F\left(\mathscr{I}^{*} \mathscr{N}_{*}\right) / F\left(K^{q}\right)=F\left(\mathscr{I}^{*} \mathscr{N}_{*} / K^{q}\right)$. Для второй спектральной последовательности этого двойного комплекса

$$
E_{2}^{i j}=F\left(\mathscr{I}^{i} H_{-j}\left(\mathscr{N}_{*}\right)\right)=0
$$

а поскольку фильтрация в нем уже регулярна, это означает, что

$$
H_{*}\left(F\left(\mathscr{I}^{*} \mathscr{N}_{*}\right) / F\left(K^{q}\right)\right)=0 .
$$

Следовательно, из точной последовательности гомологий вытекает, что вторая фильтрация в гипергомологиях вырождается в равенства $\Phi^{q} \mathbb{H}_{m}=\mathbb{H}_{m}\left(\mathcal{N}_{*} ; F\right)$.

Для описания первой фильтрации пусть $\mathscr{Z}_{m} \subset \mathscr{N}_{m}-$ ядра ( и образы) граничного оператора $\mathscr{N}_{*}$. Короткой точной последовательности

$$
0 \rightarrow \mathscr{Z}_{m+1} \rightarrow \mathscr{N}_{m+1} \rightarrow \mathscr{Z}_{m} \rightarrow 0
$$

отвечает последовательность производных функторов

$$
\cdots \rightarrow F\left(\mathscr{N}_{m+1}\right) \rightarrow F\left(\mathscr{Z}_{m}\right) \rightarrow F^{1}\left(\mathscr{Z}_{m+1}\right) \rightarrow \cdots
$$

из которой следует наличие включения $i_{m}^{0}: H_{m}\left(F\left(\mathscr{N}_{*}\right)\right) \subset F^{1}\left(\mathscr{Z}_{m+1}\right)$ и гомоморфизмов $i_{m}^{k}: F^{k}\left(\mathscr{Z}_{m}\right) \rightarrow F^{k+1}\left(\mathscr{Z}_{m+1}\right)$. Следующее предложение дает "внутреннее" описание гипергомологий $\mathbb{H}_{m}\left(\mathcal{N}_{*} ; F\right)$ и фильтрации первого типа. 


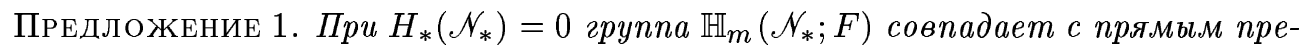
делом групп $F^{k}\left(\mathscr{Z}_{m+k}\right)$ и их гомоморфизмов $(-1)^{m+k} i_{m+k}^{k}$. Подгруппь $\Phi_{p} \mathbb{H}_{m}$ совпадают с образами в прямом пределе групп $F^{p}\left(\mathscr{Z}_{m+p}\right)$.

ДокАЗАТЕЛЬСтво. Строки $\mathscr{I}^{q} \mathscr{N}_{*}$ бикомплекса $K_{p}$ представляют собой инъективные резольвенты объектов $\mathscr{I}^{q} \mathscr{Z}_{p}$ (занумерованныев обратном порядке), поэтому имеем включение комплексов $F\left(\mathscr{I}^{*} \mathscr{Z}_{p}\right) \subset F\left(K_{p}\right)$. Стандартньй диаграммньй поиск (или рассмотрение для $F\left(K_{p}\right)$ второй спектральной последовательности с учетом регулярности для $K_{p}$ второй фильтрации), инъективность $\mathscr{I}^{q} \mathscr{Z}_{p}$ и $\mathscr{I}^{q} \mathscr{N}_{p}$ обеспечивают изоморфизм $F^{q}\left(\mathscr{Z}_{p}\right) \rightarrow H_{p-q}\left(F\left(K_{p}\right)\right)$.

Для $q$-мерного коцикла $z_{p}^{q} \in F\left(\mathscr{I}^{*} \mathscr{Z}_{p}\right)$ в силу инъективности $\mathscr{I}^{q} \mathscr{N}_{*}$ имеем $z_{p}^{q}=d^{\prime} c_{p+1}^{q}$, $c_{p+1}^{q} \in F\left(K_{p+1}\right)$, поэтому $d c_{p+1}^{q}=z_{p}^{q}+(-1)^{p+1} d^{\prime \prime} c_{p+1}^{q}$. Следовательно, при включении $F\left(K_{p}\right) \subset F\left(K_{p+1}\right)$ коцикл $z_{p}^{q}$ когомологичен $(-1)^{p} z_{p+1}^{q+1}=(-1)^{p} d^{\prime \prime} c_{p+1}^{q}$. Из стандартного же определения связывающего гомоморфизма, отвечающего короткой точной последовательности комплексов

$$
0 \rightarrow F\left(\mathscr{I}^{*} \mathscr{Z}_{p+1}\right) \rightarrow F\left(\mathscr{I}^{*} \mathscr{N}_{p+1}\right) \rightarrow F\left(\mathscr{I}^{*} \mathscr{Z}_{p}\right) \rightarrow 0
$$

следует, что коцикл $z_{p+1}^{q+1}$ определяет образ класса $\left\{z_{p}^{q}\right\} \in F^{q}\left(\mathscr{Z}_{p}\right)$ при гомоморфизме $F^{q}\left(\mathscr{Z}_{p}\right) \rightarrow F^{q+1}\left(\mathscr{Z}_{p+1}\right)$. Таким образом, фигурируюшие в предложении отображения совпадают с гомоморфизмами групп гомологий, индуцированньпи включениями $F\left(K_{p}\right) \subset F\left(K_{p+1}\right)$. Остается воспользоваться тем, что

$$
F\left(\mathscr{I}^{*} \mathscr{N}_{*}\right)=\sum_{p} F\left(K_{p}\right)=\underset{p}{\lim }\left\{F\left(K_{p}\right)\right\}
$$

и перестановочностью точного функтора $\underset{\lim }{\longrightarrow}$ операцией $H_{p}$ перехода к гомологиям [6, гл. VIII]. Предложение доказано.

Примером комплекса $\mathscr{N}_{*}$, для которого $H_{*}\left(\mathscr{N}_{*}\right)=0$, может послужить любая резольвента $0 \rightarrow \mathscr{A} \rightarrow \mathscr{N}^{*}\left(\mathscr{N}_{-p}=\mathscr{N}^{p}\right.$ при $p \geqslant 0$ и $\left.\mathscr{N}_{1}=\mathscr{N}^{-1}=\mathscr{A}\right)$. Для любого когомологического функтора $\left\{F^{q}\right\}$ в смысле Гротендика (т.е. точного $\partial$-функтора, для которого $F^{0}=F$ и $F^{q}=0$ при $q<0$, см. [7, п. 2.1]) в групше $F^{m}(\mathscr{A})$ возникает конечная фильтрация, определяемая последовательностью связьваюших гомоморфизмов $H^{m}\left(F\left(\mathscr{N}^{*}\right)\right) \rightarrow F^{1}\left(\mathscr{Z}^{m-1}\right) \rightarrow \cdots \rightarrow F^{q}\left(\mathscr{Z}_{p}\right) \rightarrow \cdots(p+q=m)$, структура которой отражает степень неацикличности членов $\mathscr{N}^{p}$ резольвенты. В случае, когда $\mathscr{N}^{*}$ - резольвента пучка $\mathscr{A}$ на топологическом пространстве $X$, определяемая покрытием $\alpha=\left\{M_{\lambda}\right\}$, пучок $\mathscr{N}^{p}$ определяется предпучком, в котором открытым множествам $U \subset X$ сопоставляются произведения по всем наборам индексов $\left(\lambda_{0}, \lambda_{1}, \ldots, \lambda_{p}\right)$ групा сечений $\mathscr{A}\left(U_{\lambda_{0}} \cap \cdots \cap U_{\lambda_{p}} \cap U\right)$ (см. [3, гл. II, п. 5.2]), $F^{q}(\mathscr{A})=H^{q}(X ; \mathscr{A})$ - функторы когомологий $X$ с коэффициентами в пучках, причем влияюшие на сложность фильтрации группы $F^{q}\left(\mathscr{N}^{p}\right)=H^{q}\left(X ; \mathscr{N}^{p}\right)$ совпадают с $p$-мерньми коцепями на нерве $|\alpha|$ покрытия $\alpha$ в системе коэффициентов, в которой симплексам $\left(\lambda_{0}, \ldots, \lambda_{i}\right) \in|\alpha|$ сопоставляются группы $H^{q}\left(U_{\lambda_{0}} \cap \cdots \cap U_{\lambda_{i}} ; \mathscr{A}\right)$ (см. [3, гл. II, п. 5.3, 5.4]). Поскольку когомологии $|\alpha|$ с указанными коэффишиентами - это члены $E_{2}^{p q}$ отвечающей резольвенте спектральной последовательности [3], фильтрация в $H^{m}(X ; \mathscr{A})$ зависит, конечно, не только от когомологий пересечений $U_{\lambda_{0}} \cap \cdots \cap U_{\lambda_{p}}$, но и от гомологического устройства покрытия $\alpha$ (т.е. комплекса $|\alpha|$ ). 
Во втором типичном частном случае составляющие комплекс $\mathscr{N}_{*}$ объекты $F$-ацикличны. В этом случае, прежде всего, $\mathbb{H}_{m}\left(\mathscr{N}_{*} ; F\right)=H_{m}\left(F\left(\mathscr{N}_{*}\right)\right)$ - гомологии комплекса $\mathscr{N}_{*}$ для функтора $F$ (гомологии $F\left(\mathscr{N}_{*}\right)$ ), поскольку для первой спектральной последовательности (всегда сходящейся из-за регулярности фильтрации) $E_{2}^{p q}=0$ при $q>0$ и $E_{2}^{p 0}=H_{-p}\left(F\left(\mathscr{N}_{*}\right)\right)$ (см. вьше). По той же причине первая фильтрация сводится к изоморфизмам $\Phi_{p} \mathbb{H}_{m}=H_{m}\left(F\left(\mathscr{N}_{*}\right)\right)$ при $p \geqslant m$. Это видно из того, что

$$
F\left(K_{p+1}\right) / F\left(K_{p}\right)=F\left(\mathscr{I}^{*} \mathscr{N}_{p+1}\right)
$$

- ацикличные при $q>0$ комплексы: $H^{q}\left(F\left(\mathscr{I}^{*} \mathscr{N}_{p+1}\right)\right)=F^{q}\left(\mathscr{N}_{p+1}\right)=0$.

Укажем "внутреннее" определение второй фильтрации. Имеем $\mathscr{B}_{p} \subset \mathscr{Z}_{p} \subset \mathscr{N}_{p}$, где $\mathscr{B}_{p}$ - образы граничного гомоморфизма. В силу $F$-ацикличности $\mathscr{N}_{p}$ точные последовательности $0 \rightarrow \mathscr{Z}_{p} \rightarrow \mathscr{N}_{p} \rightarrow \mathscr{B}_{p-1} \rightarrow 0$ при $q>1$ обеспечивают изоморфизмы $\delta: F^{q-1}\left(\mathscr{B}_{p-1}\right) \rightarrow F^{q}\left(\mathscr{Z}_{p}\right)$, поэтому включениями $\mathscr{B}_{p} \subset \mathscr{Z}_{p}$ определены гомоморфизмы $i_{p}^{q}: F^{q}\left(\mathscr{Z}_{p}\right) \rightarrow F^{q-1}\left(\mathscr{Z}_{p-1}\right)$. Из точной последовательности

$$
0 \rightarrow F\left(\mathscr{Z}_{m+1}\right) \rightarrow F\left(\mathscr{N}_{m+1}\right) \rightarrow F\left(\mathscr{B}_{m}\right) \rightarrow F^{1}\left(\mathscr{Z}_{m+1}\right) \rightarrow 0
$$

отвечающей короткой точной последовательности

$$
0 \rightarrow \mathscr{Z}_{m+1} \rightarrow \mathscr{N}_{m+1} \rightarrow \mathscr{B}_{m} \rightarrow 0
$$

вытекает наличие включения $i_{m+1}^{1}: F^{1}\left(\mathscr{Z}_{m+1}\right) \subset H_{m}\left(F\left(\mathscr{N}_{*}\right)\right)$.

ПРЕДЛОЖЕНИЕ 2. Если $\mathcal{N}_{*}$ coстоит из F-ацикличных обгектов, то вторая фильтрация в $\mathbb{H}_{m}\left(\mathscr{N}_{*} ; F\right)=H_{m}\left(F\left(\mathscr{N}_{*}\right)\right)$ определяется образами групп $F^{q}\left(\mathscr{Z}_{m+q}\right)$ при итерачиях гомоморфизмов $(-1)^{m+k} i_{m+k}^{k}, k \leqslant q$.

Ситуация оказывается заметно сложнее, чем в первом случае. Заметим, что каждьй коцикл комплекса $F\left(\mathscr{I}^{*} \mathscr{Z}_{m+k}\right)$ оказывается и циклом комплекса $F\left(K^{j}\right)$ при $j \leqslant k$. Этим определяются отображения $F^{k}\left(\mathscr{Z}_{m+k}\right) \rightarrow \Phi^{k} \mathbb{H}_{m}$. Посредством стандартного диаграммного поиска всякий цикл комплекса $F\left(K^{k}\right)$ заменяется гомологичным ему циклом, принадлежашим $F\left(\mathscr{I}^{k} \mathcal{N}_{m+k}\right)$, оказываюшимся, очевидно, $k$-мерным коциклом комплекса $F\left(\mathscr{I}^{*} \mathscr{Z}_{m+k}\right)$. Это означает, что группы $\Phi^{k} \mathbb{H}_{m}$ - эпиморфные образы групп $F^{k}\left(\mathscr{Z}_{m+k}\right)$ при включении $F\left(\mathscr{I}^{*} \mathscr{Z}_{m+k}\right) \subset F\left(\mathscr{I}^{*} \mathscr{N}_{*}\right)$. Детали доказательства см. в [8, § 2], где фильтрующие отображения оцениваются с точностью до знака. Из приведенной там схемы рассуждений прослеживается, что определенные выше эпиморфизмы с точностью до указанного в предложении 2 знака коммутируют с отображениями $i_{m+k}$ и включениями $\Phi^{k} \mathbb{H}_{m} \subset \Phi^{k-1} \mathbb{H}_{m}$.

Примером комплекса с $F$-ацикличными составляющими является дифференциальный пучок $\mathscr{N}^{*}$ на топологическом пространстве $X$, служащий прямьм образом при непрерьвном отображении $Y \rightarrow X$ вялой резольвенты пучка коэффициентов на $Y$ и используемьй для построения спектральной последовательности Лере непрерьвного отображения [3, гл. II, п. 4.17] ( $F=\Gamma_{\varphi}-\not$ фнктор сечений с носителями в семействе $\left.\varphi\right)$. Типичными примерами могут служить проективные системы $\mathscr{N}_{*}=\left\{C_{*}(\lambda)\right\}$ цепных комплексов, трактуемые как комплексы в категории проективных абелевьх групп или модулей, для функтора обратного предела $F=\lim _{\lambda}$. Соответствующие конструкции и 
спектральная последовательность применялись еще Роосом в [9], а затем и другими авторами (см. в связи с этим $[10, \S 5])$. Наконец, очевидньй интерес представляют дифференциальные пучки цепей $\mathscr{N}_{*}=\mathscr{C}_{*}(X ; \mathscr{G})$ на топологическом пространстве $X\left(F=\Gamma_{\varphi}\right.$, $\mathscr{G}$ - локально постоянные коэффициенты).

Конкретно природа обсуждаемой фильтрации выяснена пока лишь в последнем случае, где она оказывается тесно связанной с фильтрацией Зимана [11] в гомологиях, определенной сперва для конечных комплексов чисто комбинаторно, и является, очевидно, окончательной формой последней. Для вялого пучка цепей $\mathscr{N}_{*}=\mathscr{C}_{*}(X ; \mathscr{G})$ на локально компактном пространстве $X$ и паракомпактифищирующего семейства носителей $\varphi$ подгруппа $\Phi^{q} \mathbb{H}_{m}^{\varphi} \subset H_{m}^{\varphi}(X ; \mathscr{G})$ содержится в образах $H_{m}^{\varphi}(X \backslash \mathscr{A} ; \mathscr{G}) \rightarrow H_{m}^{\varphi}(X ; \mathscr{G})$ для любых подмножеств $\mathscr{A} \subset X$, размерность которых меньше $q$. Она совпадает с пересечением всех таких образов в случае, когда $X=K$ - локально конечньй клеточньй комплекс, а $\varphi$ - семейство компактных или всех замкнутых множеств. В этом случае $\Phi^{q} \mathbb{H}_{m}^{\varphi}$ также образ в $H_{m}^{\varphi}(K ; \mathscr{G})$ группы $H_{m}^{\varphi}\left(K \backslash K^{q-1} ; \mathscr{G}\right)\left(K^{q-1}-(q-1)\right.$-мерный остов $K)$; см. по этому поводу $[8, \S 3,4]$ (сушественно описание фильтрации, даваемое предложением 2).

Московский государственный университет

Поступило

им. М.В. Ломоносова

\section{СПИСОК ЦИТИРОВАННОЙ ЛИТЕРАТУРЫ}

[1] Картан А., Эйленберг С. Гомологическая алгебра. М.: ИЛ, 1960.

[2] Бредон Г.Э. Теория пучков. М.: Наука, 1988.

[3] Годеман Р. Алгебраическая топология и теория пучков. М.: ИЛ, 1961.

[4] Jensen C. U. Les foncteurs dérivés de lim et leurs applications en théorie des modules // Lect. Notes in Math. 1972. V. 254.

[5] Скляренко Е. Г. О природе гомологических умножений и двойственности // УМН. 1994. Т. 49. № 1. С. 141-198.

[6] Стинрод Н., Эйленберг С. Основания алгебраической топологии. М.: Физматгиз, 1958.

[7] Гротендик А. О некоторых вопросах гомологической алгебры. М.: ИЛ, 1961.

[8] Скляренко Е. Г. О фильтрации Зимана в гомологиях // Матем. сб. 1992. Т. 183. № 12. C. $103-116$.

[9] Roos J. E. Sur les foncteurs dérivés de $\lim _{\leftarrow}$. Applications // C. R. Acad. Sci. Paris. 1961. V. 252. P. 3702-3704.

[10] Скляренко Е. Г. Гипер(ко)гомологии для точных слева ковариантньх функторов и теория гомологий топологических пространств // УМН. 1995. Т. 50. № 3. С. 109-146.

[11] Zeeman E. C. Dihomology. III. A generalization of the Poincaré duality for manifolds // Proc. London Math. Soc. 1963. V. 13. № 49. P. 119-183. 